\title{
FIT for purpose
}

Colorectal cancer may be asymptomatic, and abdominal symptoms are a poor indicator of neoplasia. Early diagnosis is fundamental to improving survival and the current 2-weekwait (2WW) lower gastrointestinal pathway has had minimal impact on early diagnosis, as the number of cancers detected in this group is low. The detection of globin in faeces using faecal immunochemical tests (FIT) represents a significant advance from guaiac testing, because it is quantitative, thus the sensitivity and specificity for detection of colorectal cancer can be altered. This technology provides an exciting opportunity to improve cancer detection in both symptomatic and screening populations. Problems still remain, however, around the heterogenicity and standardisation of detection systems, and the most effective use of this technology.

National Institute for Health and Care Excellence (NICE) guideline NG12 was updated in 2015 to support the use of stool testing for occult blood. At the time, this raised concerns, but the predicted large increase in referrals did not materialise, presumably because the only test available was guaiac-based. DG30 supports FIT as a positive predictor of disease and suggests a $2 \mathrm{WW}$ referral in patients with low risk symptoms and a FIT level $>10 \mu \mathrm{g} / \mathrm{g}$. This opportunistic screening will inevitably increase the demand on diagnostic services, whether endoscopic or radiological, but the approach is reasonable as colorectal cancer is present in $\sim 5 \%$ of these individuals.

In the bowel cancer screening programme, FIT at a threshold of $120 \mu \mathrm{g} / \mathrm{g}$ will have replaced guaiac testing in April 2019. This threshold was chosen to mirror the current positivity of guaiac testing. The expected increased uptake, however, is likely to result in increased demand for endoscopy services in most areas, although the true effect will only be realised at rollout. There are aspirations to reduce this threshold to $20 \mu \mathrm{g} / \mathrm{g}$ and, combined with the aim to extend the screening age to start at 50 years, demand on endoscopy services will increase further.

Despite the potential benefits of FIT, current endoscopy or radiology services cannot meet this increased demand and also deliver the 62 day target to treatment. Currently, trusts are unable to fund expansion of these services and the current workforce is not sufficient to deliver the required increase. To counter the increased demand on diagnostic services, FIT could be used as a negative predictor of disease in patients with high-risk symptoms and thus reduce the number of $2 \mathrm{WW}$ referrals in patients referred on the basis of symptoms alone. This, however, would need central support to facilitate easier rejection of $2 \mathrm{WW}$ referrals and make clear where the clinical responsibility is held. Although FIT, at the limit of detection $<4 \mu \mathrm{g} / \mathrm{g}$, has a negative predictive value around $99 \%$ it may miss some tumours, and the sensitivity is improved if iron deficiency is excluded.

The bowel scope programme is extremely demanding on endoscopy services and its future has recently been the subject of a consultation process. The scientific data underpinning this service, however, is extremely robust, and the current recommendation from the UK National Screening Committee is that bowel scope will continue for the foreseeable future. If it were to be withdrawn, the large number of endoscopists delivering this service could potentially be retrained to deliver colonoscopy, but this would require a significant financial and time commitment. Further capacity to perform colonoscopy may result from implementation of the anticipated British Society of Gastroenterology guidelines on surveillance, or from gastroenterologists being released from general medical responsibilities.

FIT is an exciting technology and provides an opportunity to refine the selection of patients undergoing colonoscopy and improve early colorectal cancer detection. Despite the uncertainty in how it should be best used, there is sufficient data to support its introduction as both a positive and negative predictor of disease. Changes in practice within current diagnostic services, however, will be required to create sufficient capacity to deliver this.

WILLIAM HOWSON

Consultant, Imperial College Healthcare NHS Trust, London, UK GEOFF SMITH

Consultant, Imperial College Healthcare NHS Trust, London, UK JOHN MARTIN

Consultant, Imperial College Healthcare NHS Trust, London, UK

Address for correspondence: Dr William Howson, Imperial College Healthcare NHS Trust, The Bays, South Wharf Road, St Mary's Hospital, London W2 1NY, UK. 